Thomas Kokholm

Pricing of Traffic Light Options and other Correlation Derivatives

Finance

Research Group 


\title{
Pricing of Traffic Light Options and other Correlation Derivatives
}

\author{
Thomas Kokholm* \\ Finance Research Group, Department of Business Studies, Aarhus School of \\ Business, University of Aarhus, Fuglesangs Allé 4, DK-8210 Aarhus V, Phone: \\ +4589486693, e-mail: thko@asb.dk \\ Working paper - February 8, 2008
}

\begin{abstract}
This paper considers derivatives with payoffs that depend on a stock index and underlying LIBOR rates. A traffic light option pricing formula is derived under lognormality assumptions on the underlying processes. The traffic light option is aimed at the Danish life and pension sector to help companies stay solvent in the traffic light stress test system introduced by the Danish Financial Supervisory Authorities in 2001. Similar systems are now being implemented in several other European countries.

A pricing approach for general payoffs is presented and illustrated with simulation via the pricing of a hybrid derivative known as the EUR Sage Note. The approach can be used to price many existing structured products.
\end{abstract}

JEL classifications: C15; G13; G22; G23

Key words: LIBOR market model, traffic light option, correlation, simulation, derivatives pricing, structured products.

*Thanks to Peter Løchte Jørgensen, Rolf Poulsen, David Skovmand and Fabio Mercurio for useful suggestions. Further, the many comments received from the participants at the First Nordic Finance Network Workshop 2007, The 20th Australasian Finance and Banking Conference 2007 and The Quantitative Methods in Finance Conference 2007 have been helpful. 


\section{Introduction}

Little attention has been directed towards the pricing of derivatives with payoffs depending on both a stock index and some benchmark interest rates, even though parts of the banking community are now offering their clients the possibility to invest in such hybrid products. ${ }^{1}$ The aim of this paper is to show how European style derivatives with payoffs, depending on both a stock index and underlying LIBOR rates, can be priced, and special attention will be paid to the pricing of traffic light options first introduced in Jørgensen (2007). The traffic light option is a tailor made derivative aimed at the Danish Life and Pension (L\&P) sector to help companies stay solvent in the so-called traffic light scenarios introduced by the Danish Financial Supervisory Authorities (DFSA) in 2001. In short, the traffic light system is a supervision tool that consists of different scenarios, where both the interest rate level and stock prices fall simultaneously. L\&P companies are typically exposed in these scenarios for two reasons. First, the duration is typically much longer on the liability side than on the asset side, making the company exposed to negative shocks to interest rate levels. Second, many L\&P companies have issued guarantees on policy holder contributions, which, with the low interest rate levels today, forces the companies to invest in the stock market in order to capture the higher return here. This investment behavior exposes the companies to negative shocks of the stock market. The company is labeled in red light if its base capital cannot stay above a certain critical level (4.5\% of the pension obligations) in the scenario where interest rate levels decrease $70 \mathrm{bps}$, the stock market by $12 \%$, and real estate by $8 \%$. If this happens, the company is strictly monitored by the DFSA and forced to submit monthly solvency reports. The company is labeled in yellow light and required to submit quarterly solvency reports if it is able to stay above the critical level in the red scenario, but not in the yellow scenario, which consists of a 100 bps decrease in interest rates, $30 \%$ decrease in the stock market and a $12 \%$ decrease in real estate values. Finally, the company is "healthy" and labeled in green light if it stays above the level in the yellow scenario. In the above described scenarios, the traffic light option has a payoff. ${ }^{2}$

It is well known that when the stock market falls, many investors move their investments to "safe harbour" and invest in bonds. This drives the interest rates down, meaning that the option will be very valuable under these cir-

\footnotetext{
1 See BNP Paribas, Derivatives Handbook (2006) and Hunter and Picot (2005) for examples of hybrid products of this type. In this paper, the term hybrid derivative and correlation derivative will be used synonymously to denote products with payoffs depending on a stock index and underlying interest rates.

2 One could introduce a "real estate" payoff in the traffic light option as well, but this is not done since the real estate part of total portfolio value is normally insignificant.
} 
cumstances. A recent example where this happened was the Wall Street panic in the beginning of January 2008, where sharp drops in the US stock market drove investors to the bond market, and further led the FED to lower the federal funds rate from $4.25 \%$ to $3.50 \%$ on January $22 \mathrm{nd}$ and an extra $0.5 \%$-point on Wednesday the following week. This is an example of a scenario were the traffic light option can help pension companies remain solvent.

The model in Jørgensen (2007) is an extension of the Black-Scholes-Vasicek model, where the benchmark interest rate is determined by the dynamics of the instantaneous short rate, and a closed form solution for the price of the traffic light option is derived. The use of an instantaneous short rate process for the development in interest rates has certain drawbacks. Especially, all zero-coupon bond yields in a one-factor model are perfectly dependent, making this setup less flexible. Basing a model on instantaneous rates further introduces problems from a practical perspective, since observed market rates are quoted on a discrete time basis. Among other things, this lack of flexibility led to the development of the LIBOR market model in 1997 by Brace, Gatarek and Musiela (1997), Miltersen, Sandmann, and Sondermann (1997) and Jamshidian (1997). The LIBOR market model has been applied in several papers, but the pricing of hybrid products in a LIBOR market framework has not been studied. The market has adopted the LIBOR market model and it is now the preferred setup for many practical purposes.

This paper has two contributions: First, a closed form solution for the traffic light option price is derived, in the case where the underlying rate is some LIBOR rate. The closed form derivation is possible under lognormal distributional assumptions on the underlying processes. This closed form solution is new. Second, under more general assumptions and more advanced payoffs, one will be forced to use Monte Carlo simulation in order to price correlation derivatives. A pricing approach in these cases is presented and it is done under the spot LIBOR measure introduced in Jamshidian (1997). This is the equivalent martingale measure which corresponds to a discrete bank account as the numeraire. The method is applied to price a variant of a derivative issued by BNP Paribas called the EUR Sage Note, which has a payout similar to the ones seen in various structured products. The market for structured products has exploded in recent years. The volume of new issuances of stuctured products in the US amounted to USD 28 billion in 2003 and have risen to over 100 billion dollars in $2007 .{ }^{3}$ A major part of these structured products has a payment profile that depends on both the performance of a stock index and one or more interest rates.

In Section 2, we set up the model, and the price of the traffic light option is derived in the lognormal setting. A pricing approach for more general payoffs

3 Source: Structured Products Association. 
is also presented. Then numerical implementation of the closed form solution and with Monte Carlo simulation is performed in Section 3. The illustration of the Monte Carlo implementation is obtained via the pricing of the EUR Sage Note, but it is straightforward to apply the approach to other payoffs.

\section{Model}

In this section, a framework for modelling derivatives with payoffs depending on both an underlying LIBOR rate and an underlying stock index is defined.

It is assumed that a set of settlement dates is given,

$$
0 \leq T_{0}<T_{1}<\cdots<T_{n}
$$

This is known as the tenor structure. Later, in the Monte Carlo implementation, it is assumed that the length between two tenor dates is fixed (i.e., $\left.\tau_{j}=T_{j}-T_{j-1}=\tau\right)$ but in general $\tau_{j} \neq \tau_{i}$. The existence of a filtered prob-

ability space $\left(\Omega, \mathcal{F},\left\{\mathcal{F}_{t}\right\}_{t \geq 0}, \mathbb{P}\right)$ with the physical probability measure $\mathbb{P}$ is assumed. It is also assumed that at time zero and for each tenor date $T_{j}$, the price of a zero coupon bond maturing at that date $B\left(0, T_{j}\right)$ is given. And finally, all the standard assumptions of efficient and perfect markets are assumed. We start by introducing the LIBOR rate $L_{i}(t)$ defined by

$$
\begin{aligned}
1+\tau_{i+1} L_{i}(t) & =\frac{B\left(t, T_{i}\right)}{B\left(t, T_{i+1}\right)} \\
L_{i}(t) & =\frac{1}{\tau_{i+1}}\left(\frac{B\left(t, T_{i}\right)}{B\left(t, T_{i+1}\right)}-1\right) .
\end{aligned}
$$

$L_{i}(t)$ is the prevailing simply compounded forward rate from $T_{i}$ to $T_{i+1}$ as seen at time $t$. Let $\mathbb{Q}^{T}$ denote the equivalent martingale measure (EMM) corresponding to the numeraire $B(t, T)$. Then it is seen from (1) that the process $L_{i}$ is a martingale under the forward measure $\mathbb{Q}^{T_{i+1}}=\mathbb{Q}^{i+1}$ due to the First Fundamental Theorem of Asset Pricing. ${ }^{4}$ Assuming a continuous evolution gives rise to the LIBOR rate dynamics

$$
d L_{i}(t)=\lambda\left(t, T_{i}\right) d W^{i+1}(t)
$$

where $\lambda\left(t, T_{i}\right)$ is some (multivariate) stochastic process and $W^{i+1}$ is a (multivariate) Wiener process under $\mathbb{Q}^{i+1}$. In the lognormal LIBOR market model, the diffusion term is given by $\lambda\left(t, T_{i}\right)=L_{i}(t) \lambda_{i}(t)$ for some deterministic

$\overline{4}$ Delbaen and Schachermayer (1998). 
function $\lambda_{i}(t)$ such that the dynamics take the form

$$
d L_{i}(t)=L_{i}(t) \lambda_{i}(t) d W^{i+1}(t)
$$

In this setup, the pricing of a $T_{i}$-claim $C\left(S\left(T_{0}\right), \ldots, S\left(T_{i}\right), L_{0}\left(T_{0}\right), \ldots, L_{n}\left(T_{i}\right)\right)$ is considered, where $S$ is some stock index and $i \leq n .{ }^{5}$ We know from the Fundamental Theorem of Asset Pricing that under the assumption of no arbitrage possibilities, an EMM $\mathbb{Q}^{N}$ exists, so that the price is given by

$$
\pi(t)=N(t) \mathbb{E}^{\mathbb{Q}^{N}}\left[\frac{1}{N\left(T_{i}\right)} C\left(S\left(T_{0}\right), \ldots, S\left(T_{i}\right), L_{0}\left(T_{0}\right), \ldots, L_{n}\left(T_{i}\right)\right) \mid \mathcal{F}_{t}\right],
$$

where $N$ is a positive price process. Here the question of what numeraire $N$ to choose (or equivalently what EMM $\mathbb{Q}^{N}$ ) naturally arises. An obvious choice is to let the numeraire be the zero-coupon bond maturing at time $T_{n+1}$, since we know that $L_{n}$ is a martingale under this measure with dynamics given by (2). With this choice the problem of finding the dynamics of $S$ and all the other LIBOR rates under this measure remains. So when the payoff is more complex, it is convenient to choose another numeraire. One possibility is to use the discrete savings account, which is dealt with later in the paper. First, a price formula for the traffic light option is derived using the $T_{n+1}$ zero-coupon bond as numeraire.

\subsection{Valuation of the traffic light option under the forward measure}

In this section, the valuation of the traffic light option with $T_{n+1}$-payoff given by

$$
C\left(S\left(T_{n+1}\right), L_{n}\left(T_{n}\right)\right)=\left[\bar{S}-S\left(T_{n+1}\right)\right]^{+} \cdot\left[\bar{L}-L_{n}\left(T_{n}\right)\right]^{+}
$$

5 This is the most general payoff that can be priced by the method described in this paper. In the next section a simpler payoff is considered. 
is considered. ${ }^{6}$ Choosing the zero-coupon bond maturing at time $T_{n+1}$ as numeraire is very convenient. Inserting in (3) results in

$$
\begin{aligned}
\pi(t) & =B\left(t, T_{n+1}\right) \mathbb{E}^{n+1}\left[\frac{\left[\bar{S}-S\left(T_{n+1}\right)\right]^{+} \cdot\left[\bar{L}-L_{n}\left(T_{n}\right)\right]^{+}}{B\left(T_{n+1}, T_{n+1}\right)} \mid \mathcal{F}_{t}\right] \\
& =B\left(t, T_{n+1}\right) \\
& \times \mathbb{E}^{n+1}\left[\left[\frac{\bar{S}}{B\left(T_{n+1}, T_{n+1}\right)}-\frac{S\left(T_{n+1}\right)}{B\left(T_{n+1}, T_{n+1}\right)}\right]^{+} \cdot\left[\bar{L}-L_{n}\left(T_{n}\right)\right]^{+} \mid \mathcal{F}_{t}\right] \\
& =B\left(t, T_{n+1}\right) \mathbb{E}^{n+1}\left[\left[\bar{S}-\frac{S\left(T_{n+1}\right)}{B\left(T_{n+1}, T_{n+1}\right)}\right]^{+} \cdot\left[\bar{L}-L_{n}\left(T_{n}\right)\right]^{+} \mid \mathcal{F}_{t}\right] .
\end{aligned}
$$

Since we are working in the lognormal forward model, the LIBOR rate $L_{n}(t)$ is lognormal under its own measure. The stock index dynamics under the forward measure will depend on the instantaneous development in the zero coupon bond maturing at time $T_{n+1}$. In our setup this instantaneous development is inconvenient to model, but from the Fundamental Theorem of Asset Pricing, we know that the discounted stock index process $\frac{S(t)}{B\left(t, T_{n+1}\right)}$ is a martingale, inducing that it is driftless. It is also noticed that $\frac{S(t)}{B\left(t, T_{n+1}\right)}$ is actually the forward stock price by the no-arbitrage assumption. Assuming lognormality of the forward stock price process $\frac{S(t)}{B\left(t, T_{n+1}\right)}$ leaves us with the two stochastic differential equations

$$
\begin{aligned}
d\left(\frac{S(t)}{B\left(t, T_{n+1}\right)}\right) & =\left(\frac{S(t)}{B\left(t, T_{n+1}\right)}\right) \sigma_{t} d W_{\frac{S}{B}}^{n+1}(t) \\
d L_{n}(t) & =L_{n}(t) \lambda_{n}(t) d W_{L}^{n+1}(t)
\end{aligned}
$$

where $d W_{\frac{S}{B}}^{n+1}(t) d W_{L}^{n+1}(t)=\rho_{t} d t$ and both $\sigma_{t}$ and $\rho_{t}$ are deterministic functions of time. The volatility $\sigma_{t}$ of the discounted asset price process can be derived from market prices on ordinary European call options, since a closed

6 It may seem strange that the claim is paid at time $T_{n+1}$ since the LIBOR rate is known at time $T_{n}$. The reason for this is a technicality coming from the fact that the numeraire, under which the LIBOR rate $L_{n}$ is a martingale, is the $T_{n+1}$ zero-coupon bond, and we want to use that the time $T_{n+1}$-value of this is known at any point in time.

This construction is also observed in the market for caplets and floorlets. 
form solution given by a Black-76 formula exists.

$$
\begin{aligned}
\pi^{\text {call }}(t) & =B(t, T) \mathbb{E}^{T}\left[\frac{(S(T)-K)^{+}}{B(T, T)} \mid \mathcal{F}_{t}\right] \\
& =B(t, T) \mathbb{E}^{T}\left[\left(\frac{S(T)}{B(T, T)}-K\right)^{+} \mid \mathcal{F}_{t}\right] \\
& =S(t) N\left(d_{1}\right)-B(t, T) K N\left(d_{2}\right)
\end{aligned}
$$

where

$$
\begin{aligned}
d_{1} & =\frac{\ln \frac{S_{t}}{B(t, T) K}+\frac{1}{2} \sigma_{S}^{2}}{\sigma_{S}} \\
d_{2} & =\frac{\ln \frac{S_{t}}{B(t, T) K}-\frac{1}{2} \sigma_{S}^{2}}{\sigma_{S}}
\end{aligned}
$$

and

$$
\sigma_{S}^{2}=\int_{t}^{T} \sigma_{u}^{2} d u
$$

By assuming lognormality of the forward stock price process, the volatility of this process can be derived from quoted prices by inversion of (7).

The main result of this section and the first contribution in the paper is given in the Proposition below, where a closed form solution for the price of the traffic light option is stated.

Proposition 1 Under the assumptions in this section the time $t$ value of the traffic light option with $T_{n+1}$-payoff (4) is given by

$$
\begin{aligned}
& \pi\left(S(t), L_{n}(t), t ; \rho_{S L}\right) \\
& =S(t) L_{n}(t)\left[\widetilde{S} \cdot \widetilde{L} \cdot M\left(\frac{\ln \widetilde{S}-\mu_{x}}{\sigma_{x}}, \frac{\ln \widetilde{L}-\mu_{y}}{\sigma_{y}} ; \rho_{S L}\right)\right. \\
& -\widetilde{L} \cdot M\left(\frac{\ln \widetilde{S}-\mu_{x}}{\sigma_{x}}-\sigma_{x}, \frac{\ln \widetilde{L}-\mu_{y}}{\sigma_{y}}-\rho_{S L} \sigma_{x} ; \rho_{S L}\right) \\
& -\widetilde{S} \cdot M\left(\frac{\ln \widetilde{S}-\mu_{x}}{\sigma_{x}}-\rho_{S L} \sigma_{y}, \frac{\ln \widetilde{L}-\mu_{y}}{\sigma_{y}}-\sigma_{y} ; \rho_{S L}\right) \\
& \left.+e^{\sigma_{x y}} \cdot M\left(\frac{\ln \widetilde{S}-\mu_{x}}{\sigma_{x}}-\rho_{S L} \sigma_{y}-\sigma_{x}, \frac{\ln \widetilde{L}-\mu_{y}}{\sigma_{y}}-\rho_{S L} \sigma_{x}-\sigma_{y} ; \rho_{S L}\right)\right],
\end{aligned}
$$


where

$$
\begin{aligned}
\widetilde{S} & =\frac{\bar{S} B\left(t, T_{n+1}\right)}{S(t)} & \sigma_{x}^{2} & =\int_{t}^{T_{n+1}} \sigma_{s}^{2} d s \\
\widetilde{L} & =\frac{\bar{L}}{L_{n}(t)} & \sigma_{y}^{2} & =\int_{t}^{T_{n}} \lambda_{n}(s)^{2} d s \\
\mu_{x} & =-\frac{1}{2} \int_{t}^{T_{n+1}} \sigma_{s}^{2} d s & \sigma_{x y} & =\int_{t}^{T_{n}} \sigma_{s} \lambda_{n}(s) \rho_{s} d s \\
\mu_{y} & =-\frac{1}{2} \int_{t}^{T_{n}} \lambda_{n}(s)^{2} d s & \rho_{S L} & =\frac{\sigma_{x y}}{\sigma_{x} \sigma_{y}},
\end{aligned}
$$

and $M(\cdot, \cdot ; \rho)$ is the standardized bivariate normal distribution function with correlation coefficient $\rho$.

Proof of the Proposition can be found in Appendix A.

In the traffic light option pricing formula, the bivariate normal distribution has to be evaluated. Several routines exist for this and the one used in this paper can be downloaded from the MatLab central file exchange. ${ }^{7}$

\subsection{Valuation of general correlation derivatives under the spot measure}

It is well known that the drift of any asset under the risk neutral measure equals the instantaneous risk free rate $r_{t}$, and often dynamics of the form

$$
d S_{t}=r_{t} S_{t} d t+\sigma_{t} S_{t} d W_{S}^{\mathbb{Q}}(t)
$$

is assumed, with corresponding time $t$ value $S_{t}=S_{0} e^{\int_{0}^{t}\left(r_{s}-\frac{1}{2} \sigma_{s}^{2}\right) d s+\int_{0}^{t} \sigma_{s} d W_{S}^{\mathbb{Q}}(s)}$. However, this is not practical since it involves instantaneous rates, and it is more tractable sticking to the LIBOR rates. It is more convenient to find some discrete rate version of the dynamics (9). Instead of using the continuously compounded bank account as the numeraire, the discretely compounded analog introduced by Jamshidian (1997) can be used. The discrete bank account can be represented by a rolling over strategy. Start with one dollar at time 0 and invest in $\frac{1}{B\left(0, T_{0}\right)}$ zero coupon bonds maturing at time $T_{0}$ paying $\frac{1}{B\left(0, T_{0}\right)}$. Invest this amount in bonds which mature at the next tenor date $T_{1}$ and continue this strategy until time $t$. The value of this self financing strategy

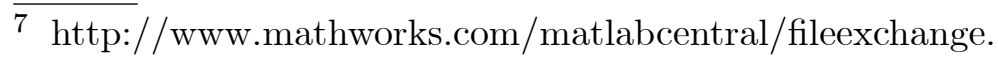


at any time $t$ is given by

$$
\begin{aligned}
B^{d}(t) & =\frac{B\left(t, T_{i(t)}\right)}{B\left(0, T_{0}\right)} \prod_{j=0}^{i(t)-1} \frac{B\left(T_{j}, T_{j}\right)}{B\left(T_{j}, T_{j+1}\right)} \\
& =\frac{B\left(t, T_{i(t)}\right)}{B\left(0, T_{0}\right)} \prod_{j=0}^{i(t)-1}\left(1+\tau_{j+1} L_{j}\left(T_{j}\right)\right),
\end{aligned}
$$

where $i(t)=\inf \left\{k \mid T_{k-1} \leq t<T_{k}\right\}$. It is the value of the trading strategy at time $T_{i(t)}$, which is then discounted back to time $t$ by multiplying with $B\left(t, T_{i(t)}\right)$. For $T_{0}=0$ and $t=T_{k}$ for some $k$, the discrete bank account reduces to $B^{d}\left(T_{k}\right)=\prod_{j=0}^{k-1}\left(1+\tau_{j+1} L_{j}\left(T_{j}\right)\right)$.

Assuming that the bond prices have dynamics

$$
d B\left(t, T_{j}\right)=B\left(t, T_{j}\right)\left(a\left(t, T_{j}\right) d t+b\left(t, T_{j}\right) d W(t)\right)
$$

under some underlying probability measure, then using Itô's lemma on $B^{d}(t)$ gives

$$
d B^{d}(t)=B^{d}(t)\left(a\left(t, T_{i(t)}\right) d t+b\left(t, T_{i(t)}\right) d W(t)\right) .
$$

Again, it is seen that the dynamics involve instantaneous drift and diffusion terms from the bond dynamics. Modelling this can be avoided by letting the claims that have to be priced be tied to the settlement dates of the LIBOR rates, which is not a restrictive assumption.

Determining the discretely compounded analog of the asset price process directly reveals

$$
S_{t}=S_{0} \frac{B\left(t, T_{i(t)}\right)}{B\left(0, T_{0}\right)} \prod_{j=0}^{i(t)-1}\left(1+\tau_{j+1} L_{j}\left(T_{j}\right)\right) e^{\int_{0}^{t} \sigma_{s} d W_{S}^{d}(s)-\frac{1}{2} \int_{0}^{t} \sigma_{s}^{2} d s} .
$$

Using the discrete bank account as numeraire, The First Fundamental Theorem of Asset Pricing dictates that there exists an equivalent measure $\mathbb{Q}^{d}$, so the discounted asset price process $\frac{S(t)}{B^{d}(t)}$ is a martingale under $\mathbb{Q}^{d 8}$

$$
\frac{S(t)}{B^{d}(t)}=\mathbb{E}^{d}\left[\frac{S(T)}{B^{d}(T)} \mid \mathcal{F}_{t}\right]
$$

and from (10) and (13) it is seen that the defined asset price process discounted $\frac{S(t)}{B^{d}(t)}$ (by construction) has the martingale property.

8 This measure will be called the spot LIBOR measure from now on, or simply just the spot measure. 
When pricing under the spot LIBOR measure, the dynamics of the LIBOR rates $L_{i}$ for $i=1, \ldots, n$ have to be found in order to calculate the expectation

$$
\pi(t)=B^{d}(t) \mathbb{E}^{d}\left[\frac{1}{B^{d}\left(T_{i}\right)} C\left(S\left(T_{0}\right), \ldots, S\left(T_{i}\right), L_{0}\left(T_{0}\right), \ldots, L_{n}\left(T_{i}\right)\right) \mid \mathcal{F}_{t}\right] .
$$

These dynamics are derived in Jamshidian (1997).

Proposition 2 (Dynamics of the LIBOR rates under the spot LIBOR measure) ${ }^{9}$

The dynamics of the LIBOR rates in the lognormal forward LIBOR model under the spot LIBOR measure is given by

$$
d L_{i}(t)=L_{i}(t) \sum_{j=i(t)}^{i} \frac{\tau_{j+1} L_{j}(t) \rho_{i, j} \lambda_{j}(t)}{1+\tau_{j+1} L_{j}(t)} \lambda_{i}(t) d t+L_{i}(t) \lambda_{i}(t) d W_{i}^{d}(t),
$$

where $W_{i}^{d}$ is a Wiener process under the spot LIBOR measure $\mathbb{Q}^{d}$, and $\rho_{i, j}$ is the correlation coefficient between the Wiener processes $W_{i}^{d}$ and $W_{j}^{d}$.

Now claims contingent on both a stock index and LIBOR rates can be priced.

Notice how the model is only completely determined on the tenor dates of the LIBOR rates. A discretization is needed between tenor dates. This can be seen from equation (14), where the time $t$ price depends on the discrete bank account at time $t$. If the time $t$ price of the derivative is needed, and since we do not want to involve the instantaneous development in the discrete bank account, a simple linear interpolation between the two nearest tenor dates is suggested to get $B^{d}(t){ }^{10}$

\subsection{Instantaneous volatilities and correlation}

When evaluating equation (14), the simultaneous distribution of the various stochastic variables under the expectation is needed, and this is in general not possible to find analytically, meaning that the evolution in the corresponding processes has to be implemented by simulation. Here, the instantaneous correlations between the different LIBOR rates and the stock index are a fundamental issue, when the simulation is implemented. The correlation matrix

$\overline{9}$ Here, the Proposition is stated in the 1-dimensional version. For a version where all the individual LIBOR rates are allowed to be driven by multivariate Browninan components and a complete proof, the reader is refered to Jamshidian (1997). A nice sketch of the proof is given in Glasserman (2004).

10 This is also suggested in Jamshidian (1997). 
stems from

$$
\left[\begin{array}{c}
d W_{1}^{d}(t) \\
\cdot \\
\cdot \\
d W_{n}^{d}(t) \\
d W_{S}^{d}(t)
\end{array}\right]\left[d W_{1}^{d}(t) \cdots d W_{n}^{d}(t) d W_{S}^{d}(t)\right]=\left[\begin{array}{ccccc}
1 & \rho_{1,2} & \cdots & \rho_{1, n} & \rho_{1, S} \\
\rho_{2,1} & 1 & \cdot & \cdot & \rho_{2, S} \\
\cdot & \cdot & 1 & \cdot & \cdot \\
\cdot & & \cdot \\
\rho_{n, 1} & \cdot & \cdot & 1 & \rho_{n, S} \\
\rho_{S, 1} & \cdot & \cdot & \rho_{S, n} & 1
\end{array}\right] d t
$$

where the correlations generally can be stochastic processes.

In the implementation, correlations between the LIBOR rates are described by deterministic functions depending on the length between the corresponding tenor dates, $T_{i}, T_{j}$. With correlations involving the stock index, the dependence is on the length between time $t$ and the corresponding tenor date of the LIBOR rate $T_{i}$.

For our purpose, it is satisfactory to specify the instantaneous volatility of the LIBOR rates following Rebonato (2002) p. 159 as

$$
\lambda_{i}(t)=f\left(T_{i}-t\right) g(T)
$$

and he argues that the functional form of $f$

$$
f\left(T_{i}-t\right)=\left(a+\left(T_{i}-t\right) b\right) e^{-\left(T_{i}-t\right) c}+d
$$

is flexible enough to capture desirable criteria such as being hump shaped. ${ }^{11}$

The instantaneous correlation matrix between the LIBOR rates should fulfill four criteria. Namely:

(1) Symmetry: $\rho_{i, j}=\rho_{j, i} \forall i, j$.

(2) It should be positive semidefinite $\left(x^{T} \rho x \geq 0 \forall x \in \mathbb{R}^{\mathbb{N}}\right)$.

(3) 1 on the diagonal $\left(\rho_{i, i}=1\right)$.

(4) Entries in the interval $[-1,1]$.

Further, I will model it as a time homogeneous function for $T_{i}, T_{j}>t$ and $i \neq j$. A simple correlation function that satisfies the requirements above is

$$
\rho_{i, j}(t)=e^{-\beta\left|T_{i}-T_{j}\right|},
$$

where $\beta>0$.

The only missing aspect now is how to specify the volatility of the stock index, and how it correlates with the LIBOR rates. For simplicity, I will let the

\footnotetext{
${ }^{11}$ Rebonato (2002) p. 167.
} 


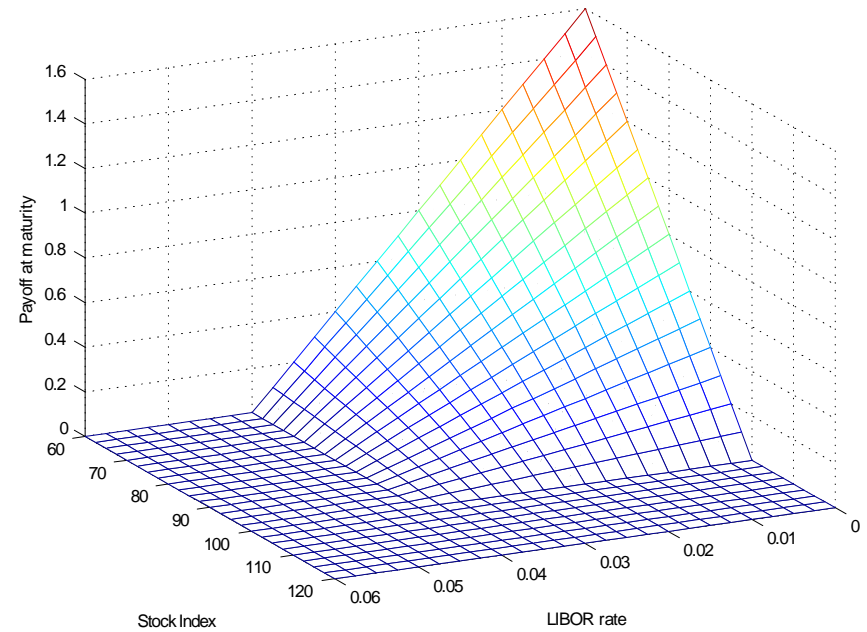

Fig. 1. The payout of the Traffic Light Option with $\bar{S}=100$ and $\bar{L}=0.04$.

volatility be constant $\left(\sigma_{s}=\sigma\right)$. It is reasonable to let the Wiener process for the stock index $W_{S}^{d}(t)$ be correlated most with the LIBOR rates with shortest distance to maturity $T_{i}-t$. A convenient form is

$$
\rho_{S, i}(t)=\frac{1-\exp \left(-\frac{\alpha}{\left(t-T_{i}-\gamma\right)}\right)}{1+\exp \left(-\frac{\alpha}{\left(t-T_{i}-\gamma\right)}\right)}=\tanh \left(\frac{\alpha}{2\left(t-T_{i}-\gamma\right)}\right),
$$

where $\gamma>0$. Positive values of $\alpha$ give rise to negative correlations and vice versa. This specific choice of function ensures correlations between -1 and 1 , and if more flexibility is needed, additional parameters can be included inside the brackets in (19). Of course, for $T_{i}<t$ the LIBOR rate has matured and the correlation is set to zero.

\section{Numerical Implementation}

Valuation of (14) is done in this section, and in the first part the $T_{n+1}$-claim given by

$$
C\left(S\left(T_{n+1}\right), L_{n}\left(T_{n}\right)\right)=\left[\bar{S}-S\left(T_{n+1}\right)\right]^{+} \cdot\left[\bar{L}-L_{n}\left(T_{n}\right)\right]^{+}
$$

is considered. This resembles the payout in Jørgensen (2007) and is illustrated in Figure 1. The only difference is that the payout is tied to a LIBOR rate instead of some benchmark interest rate depending on the instantaneous short rate process. Notice, also, that since the LIBOR rate sets 6 months prior to expiry, the traffic light option reduces to a plain vanilla equity put option the final 6 months. 


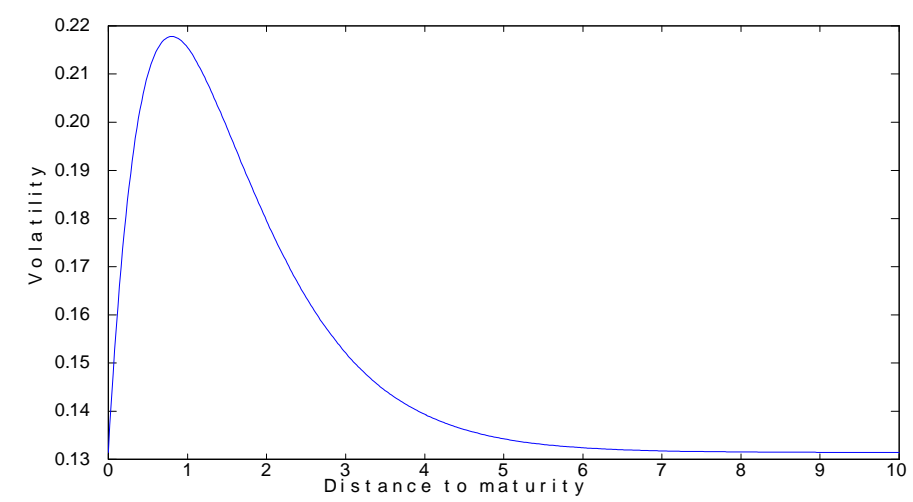

Fig. 2. The instantaneous LIBOR rate volatility as a function of $T_{i}-t$.

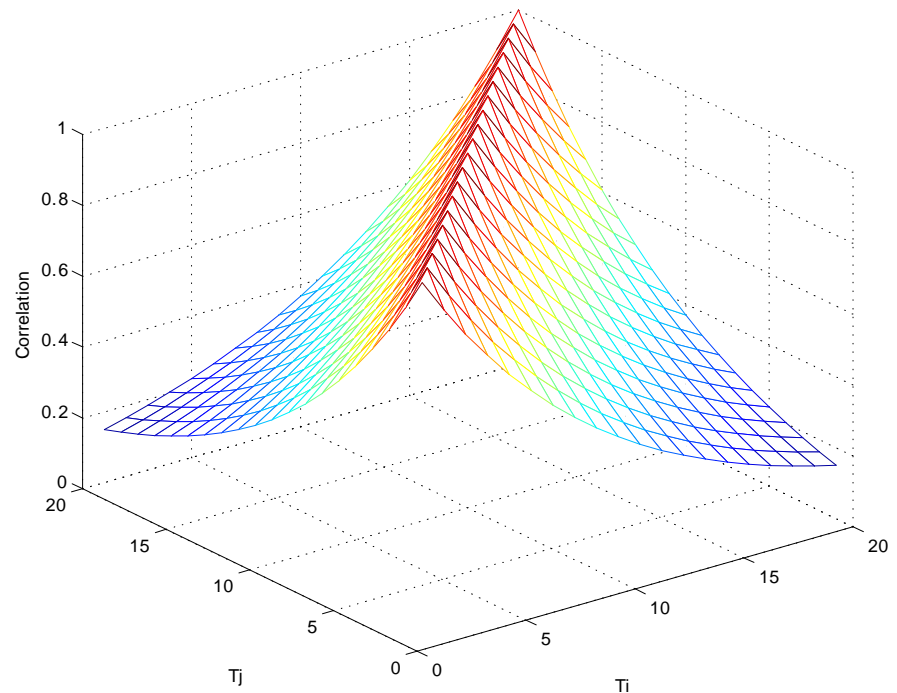

Fig. 3. Here the correlations between the LIBOR rates are illustrated for $\beta=0.1$.

The volatility structure of the LIBOR rates is assumed to have the form

$$
\begin{aligned}
\lambda_{i}(t) & =f\left(T_{i}-t\right) \\
& =\left(a+\left(T_{i}-t\right) b\right) e^{-\left(T_{i}-t\right) c}+d
\end{aligned}
$$

with parameters taken from Brigo and Mercurio (2006) p. 320

$$
a=0, \quad b=0.29342753, \quad c=1.25080230, \quad d=0.13145869,
$$

and it is illustrated in Figure 2.

Let the coefficient in (18) be given by $\beta=0.1$ as illustrated in Figure 3.

What the correct values are for the $\alpha$ and $\gamma$ parameters in the function for measuring the correlation between the stock index and the LIBOR rates 


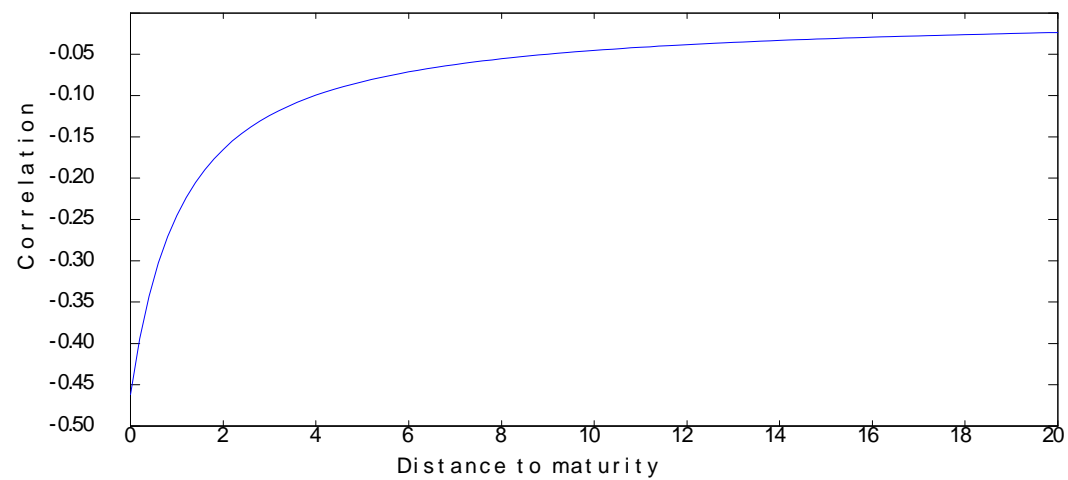

Fig. 4. Here the correlation between the stock index and the LIBOR rates as in equation (19) is depicted with $\gamma=\alpha=1$.

in equation (19) is unclear, but setting $\alpha$ and $\gamma$ equal to one yield realistic levels for the correlation as seen in Figure 4. Table 1 illustrates instantaneous correlation values as a function of both $\alpha, \gamma$ and the distance to maturity $T_{i}-t$ of the corresponding LIBOR rate. The first observation is that the correlation is a decreasing function of distance to maturity for this particular choice of correlation function. This is also a reasonable property. It is the rate maturing nearest from now that reacts most to the market information also driving the stock market. The $\alpha$ parameter controls the level of the correlation and higher absolute values of this parameter increases the absolute correlation across maturities, though not in a parallel way. The $\gamma$ parameter controls the curvature of the function. This is also clear from looking at the table, where it is seen that the absolute decrease in correlation as distance to maturity increases is highest for small values of $\gamma$. Or loosely stated, the starting point of the function in Figure 4 shifts closer to zero for higher $\gamma$ values.

\subsection{Pricing of The Traffic Light Option with Proposition 1}

In Figure 5, the price of the traffic light option is found with Proposition 1 , and depicted as a function of the stock price and the LIBOR rate at time zero. In Figure 6, the influence of the correlation on the price is illustrated. All parameters are set as mentioned under the Figures and the instantaneous LIBOR rate volatility in the calculations is specified as in Figure 2.

From Figure 5, it is seen how the traffic light option value is a decreasing function of both the stock index price and the initial value of the LIBOR rate, which was also expected from the specification of the payout function.

Further, it is seen that the option value converges towards zero, when the underlying variables move further out-of-the money, and the value is highest for the underlying variables deep in-the money. Figure 6 also shows (not sur- 


\begin{tabular}{|c|c|c|c|c|c|c|c|}
\hline & & \multicolumn{6}{|c|}{$\gamma$} \\
\hline & $\alpha$ & 0.0005 & 0.25 & 0.5 & 1 & 2 & 3 \\
\hline \multirow[t]{7}{*}{$T_{i}-t=0$} & -5.0 & 1.00 & 1.00 & 1.00 & 0.99 & 0.85 & 0.68 \\
\hline & -2.0 & 1.00 & 1.00 & 0.96 & 0.76 & 0.46 & 0.32 \\
\hline & -0.5 & 1.00 & 0.76 & 0.46 & 0.24 & 0.12 & 0.08 \\
\hline & 0.0 & 0.00 & 0.00 & 0.00 & 0.00 & 0.00 & 0.00 \\
\hline & 0.5 & -1.00 & -0.76 & -0.46 & -0.24 & -0.12 & -0.08 \\
\hline & 2.0 & -1.00 & -1.00 & -0.96 & -0.76 & -0.46 & -0.32 \\
\hline & 5.0 & -1.00 & -1.00 & -1.00 & -0.99 & -0.85 & -0.68 \\
\hline \multirow[t]{7}{*}{$T_{i}-t=2.5$} & -5.0 & 0.76 & 0.72 & 0.68 & 0.61 & 0.50 & 0.42 \\
\hline & -2.0 & 0.38 & 0.35 & 0.32 & 0.28 & 0.22 & 0.18 \\
\hline & -0.5 & 0.10 & 0.09 & 0.08 & 0.07 & 0.06 & 0.05 \\
\hline & 0.0 & 0.00 & 0.00 & 0.00 & 0.00 & 0.00 & 0.00 \\
\hline & 0.5 & -0.10 & -0.09 & -0.08 & -0.07 & -0.06 & -0.05 \\
\hline & 2.0 & -0.38 & -0.35 & -0.32 & -0.28 & -0.22 & -0.18 \\
\hline & 5.0 & -0.76 & -0.72 & -0.68 & -0.61 & -0.50 & -0.42 \\
\hline \multirow[t]{7}{*}{$T_{i}-t=7.5$} & -5.0 & 0.32 & 0.31 & 0.30 & 0.29 & 0.26 & 0.23 \\
\hline & -2.0 & 0.13 & 0.13 & 0.12 & 0.12 & 0.10 & 0.10 \\
\hline & -0.5 & 0.03 & 0.03 & 0.03 & 0.03 & 0.03 & 0.02 \\
\hline & 0.0 & 0.00 & 0.00 & 0.00 & 0.00 & 0.00 & 0.00 \\
\hline & 0.5 & -0.03 & -0.03 & -0.03 & -0.03 & -0.03 & -0.02 \\
\hline & 2.0 & -0.13 & -0.13 & -0.12 & -0.12 & -0.10 & -0.10 \\
\hline & 5.0 & -0.32 & -0.31 & -0.30 & -0.29 & -0.26 & -0.23 \\
\hline
\end{tabular}

Table 1

The instantaneous correlation between the stock index and the LIBOR rates for various values of $\gamma, \alpha$ and for three different values of distance to maturity of the rates.

prisingly) how the price is increasing in correlation. With higher correlation, there is a higher probability of realizing simultaneous drops in both the stock index and the LIBOR rate which reflects in a higher option price. It is clearly confirmed from the Figure, how the correlation choice is a very important issue when the traffic light option is priced. The difference between the highest and the lowest price is as much as $260 \%$ of the zero-correlation price.

In Table 2, the dependence of the price of an "at the money" traffic light 


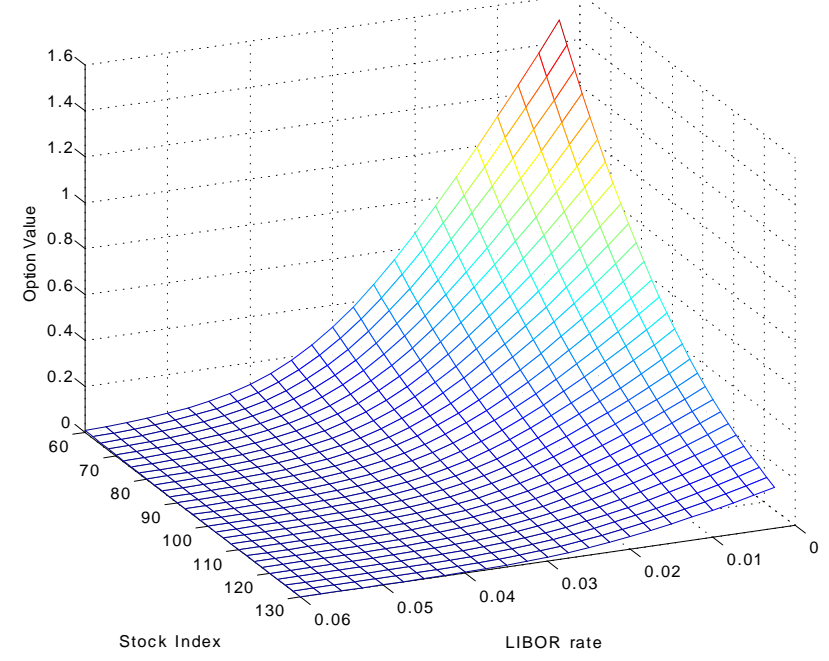

Fig. 5. Here the parameter values are $\bar{S}=100, \bar{L}=0.04, T_{n+1}=3, \rho=-0.5$, $\sigma_{s}=\sigma=0.2$ and the term structure is assumed flat equal to the intial LIBOR rate.

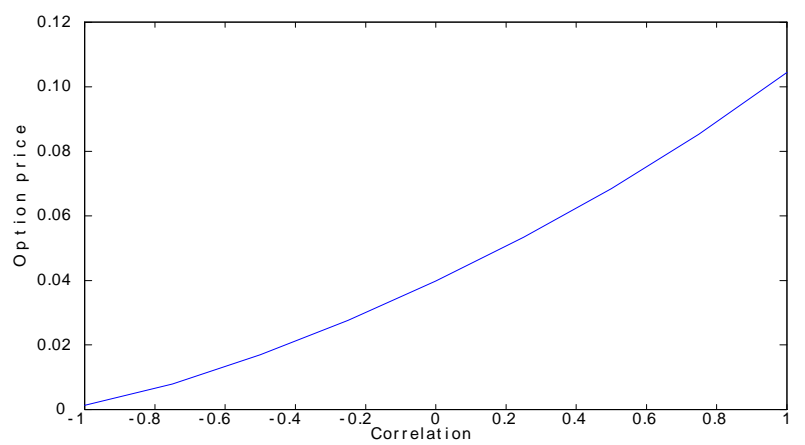

Fig. 6. The traffic light option price as a function of the correlation with $S_{0}=100$, $L_{n}(0)=0.04, \bar{S}=100, \bar{L}=0.04, T_{n+1}=3, \sigma_{s}=\sigma=0.2$ and $B\left(0, T_{n+1}\right)=0.8890$.

option is shown with respect to the instantaneous correlation and time to maturity of the option.

For example, when time to maturity is 3 years, then it means that the underlying LIBOR rate matures after $2 \frac{1}{2}$ years. From the Table, the same relationship as depicted above is seen: The correlation has a positive effect on the price of the option. With respect to time to maturity, it is seen that the price reaches its maximum somewhere between 5 and 15 years for various correlation levels, and as time to maturity converges towards infinity, the price converges towards zero. This hump-shaped behavior as a function of time to maturity is also shared with individual floorlet end put option prices in this model, so the fact that it is seen with respect to the traffic light option is not surprising. 


\begin{tabular}{|c|rrrrrrrrr|}
\cline { 2 - 10 } \multicolumn{1}{c|}{} & \multicolumn{8}{c|}{$T_{n+1}$} \\
\hline$\rho$ & 1 & 2 & 3 & 5 & 10 & 15 & 20 & 30 & 100 \\
\hline-1.00 & 0.201 & 0.166 & 0.129 & 0.095 & 0.054 & 0.030 & 0.016 & 0.005 & 0.000 \\
-0.75 & 0.402 & 0.657 & 0.786 & 0.930 & 1.051 & 1.031 & 0.953 & 0.747 & 0.057 \\
-0.50 & 0.643 & 1.304 & 1.687 & 2.113 & 2.515 & 2.536 & 2.385 & 1.905 & 0.149 \\
-0.25 & 0.922 & 2.069 & 2.760 & 3.518 & 4.243 & 4.299 & 4.051 & 3.237 & 0.252 \\
0.00 & 1.234 & 2.939 & 3.978 & 5.108 & 6.182 & 6.261 & 5.891 & 4.689 & 0.355 \\
0.25 & 1.578 & 3.908 & 5.335 & 6.875 & 8.319 & 8.408 & 7.890 & 6.243 & 0.456 \\
0.50 & 1.956 & 4.984 & 6.842 & 8.834 & 10.675 & 10.759 & 10.064 & 7.911 & 0.552 \\
0.75 & 2.371 & 6.181 & 8.524 & 11.022 & 13.294 & 13.353 & 12.439 & 9.687 & 0.632 \\
1.00 & 2.827 & 7.534 & 10.444 & 13.526 & 16.256 & 16.219 & 14.990 & 11.480 & 0.683 \\
\hline
\end{tabular}

Table 2

Traffic light option prices 100 for various parameter values of the instantaneous correlation and time to maturity. The rest of the parameters are set to: $S_{0}=\bar{S}=$ $100, L_{n}(0)=\bar{L}=0.04, \tau=0.5, \sigma_{s}=0.2$ and the LIBOR rate volatility as in Figure 2. The initial term structure is assumed flat.

\subsection{Pricing with simulation}

In practice it is possible to price any European type $T_{i}$-payoff given by $C\left(S\left(T_{0}\right), \ldots, S\left(T_{i}\right), L_{0}\left(T_{0}\right), \ldots, L_{n}\left(T_{i}\right)\right)$ by simulation. In this section a multifactor model is implemented. ${ }^{12}$ Illustration of the model is performed via the pricing of a hybrid derivative issued by BNP Paribas and known as the EUR Sage Note with a maturity of $T_{n}=15$ years and with a yearly coupon payment given by

$C\left(S_{T_{i}}, L_{T_{i}+2}\left(T_{i}\right), L_{T_{i}+10}\left(T_{i}\right)\right)=\min \left\{\max \left\{k_{i}\left(L_{T_{i}+10}-L_{T_{i}+2}\right), 0.01\right\}, 0.08\right\}$,

where the gearing $k_{i}$ is high if the EuroStoxx 50 price index performs well, ben-

efitting investors who believe that the equity market will rise. ${ }^{13}$ The gearing is given by the relation

$$
k_{i}=\left\{\begin{array}{cc}
2 & S_{T_{i}}<0.95 S_{0} \\
4 & 0.95 S_{0} \leq S_{T_{i}}<2 S_{0} \\
8 & S_{T_{i}} \geq 2 S_{0}
\end{array} .\right.
$$

$\overline{12}$ A total of $n+1$ factors drive the model here.

13 This derivative is described in BNP Paribas (2006), although with a slightly different coupon. There it is the spread between two CMS rates that are geared by $k_{i}$. 
The coupon is the spread between a long and a short LIBOR rate geared by $k_{i}$, floored by $1 \%$ and capped by $8 \%$. The rationale underlying an investment in a product like this is that the gearing is higher than what can be achieved with a normal steepener for the same price if the equity market rises substantially. Payments similar to this form is being increasingly used in the construction of structured products. The pricing is performed under the spot LIBOR measure.

In a simulation of the LIBOR rates, the first choice is to fix a time grid of future time points over which to simulate $0=t_{0}<t_{1}<\cdots<t_{m}<t_{m+1}$. In this time grid, it is convenient to let the tenor dates $T_{0}<T_{1}<\cdots<T_{n}$ be a subset. Further, by letting the time difference between two simulation points be constant $\left(t_{j+1}-t_{j}=\delta\right)$, the notation is reduced.

Simulating the LIBOR rates with an Euler scheme on $\log \widehat{L}_{i}$ results in ${ }^{14}$

$$
\widehat{L}_{i}\left(t_{j+1}\right)=\widehat{L}_{i}\left(t_{j}\right) \cdot \exp \left\{\left(\mu_{i}\left(t_{j}\right)-\frac{1}{2} \lambda_{i}\left(t_{j}\right)^{2}\right) \delta+\sqrt{\delta} \lambda_{i}\left(t_{j}\right) Z_{j+1}\right\},
$$

with

$$
\mu_{i}\left(t_{j}\right)=\sum_{l=i\left(t_{j}\right)}^{i} \frac{\tau_{l+1} \widehat{L}_{l}\left(t_{j}\right) \rho_{i, l} \lambda_{l}\left(t_{j}\right)}{1+\tau_{l+1} \widehat{L}_{l}\left(t_{j}\right)} \lambda_{i}\left(t_{j}\right),
$$

and $Z_{1}, \ldots, Z_{m+1}$ independent $N(0,1)$ random variables. In the equations above, the hats have been added to clarify that the continuous LIBOR rates have been discretized. The simulation is initialized with (1) by setting

$$
\widehat{L}_{i}(0)=\frac{1}{\tau_{i+1}}\left(\frac{B\left(0, T_{i}\right)}{B\left(0, T_{i+1}\right)}-1\right), \quad i=1, \ldots, n .
$$

The value of the bank account at maturity $T_{n}$ can be derived from the simulation of the LIBOR rates using (10). The final variable to simulate is the stock price given by $S\left(T_{n}\right)=S_{0} B^{d}\left(T_{n}\right) e^{\int_{0}^{T_{n}} \sigma_{s} d W_{S}^{d}(s)-\frac{1}{2} \int_{0}^{T_{n}} \sigma_{s}^{2} d s}$, where $X\left(T_{n}\right)=S_{0} \cdot e^{\int_{0}^{T_{n}} \sigma_{s} d W_{S}^{d}(s)-\frac{1}{2} \int_{0}^{T_{n}} \sigma_{s}^{2} d s}$ can be simulated with the scheme

$$
\widehat{X}\left(t_{j+1}\right)=\widehat{X}\left(t_{j}\right) \cdot \exp \left\{-\frac{1}{2} \sigma_{s}^{2} \delta+\sqrt{\delta} \sigma_{s} Z_{j+1}\right\} .
$$

And finally, correlation is achieved by simulating at every time step from an $(n+1)$-dimensional normal distribution with correlation matrix (16), and then use these normal variables in the discretizations above.

By the use of the discretizations above, the time zero price of the derivative

$$
\pi(0)=\sum_{i=1}^{15} \mathbb{E}^{d}\left[\frac{1}{B^{d}\left(T_{i}\right)} C\left(S_{T_{i}}, L_{T_{i}+2}\left(T_{i}\right), L_{T_{i}+10}\left(T_{i}\right)\right)\right]
$$

can then be approximated with Monte Carlo simulation.

\footnotetext{
${ }^{14}$ See Glasserman (2004) for more details.
} 


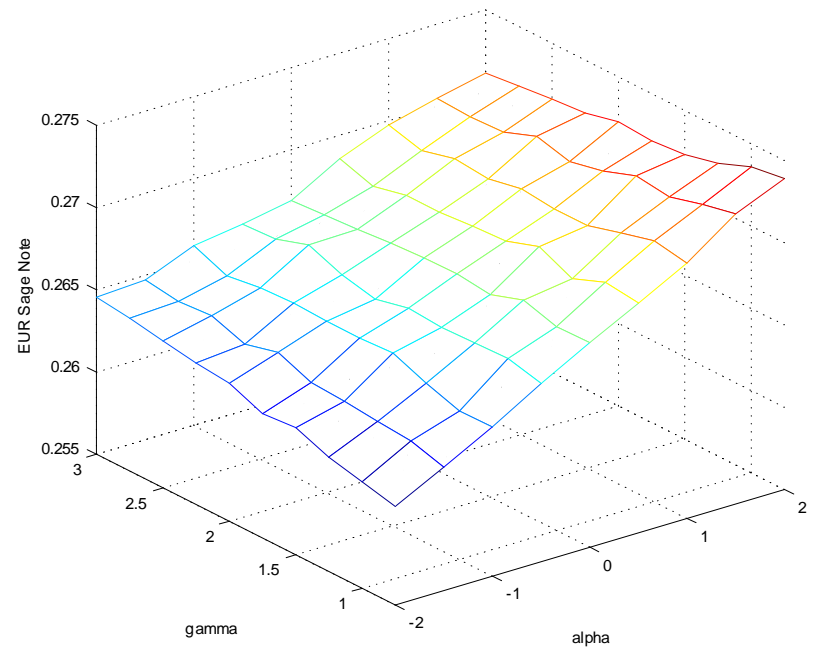

Fig. 7. The value of the hybrid derivative as a function of $\alpha$ and $\gamma$. The parameters are: $S_{0}=100, \tau=\frac{1}{2}, \delta=\frac{1}{4}, \sigma_{s}=0.2, \beta=0.1$, the LIBOR rate volatilities as in Figure 2 and the initial term structure is assumed flat with all the LIBOR rates equal to 0.04 .

In Figure 7, a plot of the hybrid derivative price is graphed as a function of the parameters $\alpha$ and $\gamma$ controlling the correlations. In the simulation, the time intervals between the tenor dates are set constant equal to one half year $\left(\tau_{i}=0.5\right)$ and the time step used in the Euler discretization is a quarter of a year $(\delta=0.25) .{ }^{15}$ Finally, each point in the graph is calculated with 200, 000 replications. The value of the derivative is highest for high values of $\alpha$ and low values of $\gamma$ when $\alpha>0$. This is explained with Table 1 , where it is seen that low values of $\gamma$ induce that the stock index correlates (in absolute value) more with the LIBOR rate in the short end. For positive values of $\alpha$, this results in negative correlations. This in turn induces ceteris paribus that the probability is higher of observing high returns on the stock market in combination with a greater decrease in rates in the short end than in the long end. ${ }^{16}$ All together, this results in higher expected coupon payments on the EUR Sage Note giving rise to a higher price. For $\alpha<0$, the effect of $\gamma$ is opposite, since a low value of $\gamma$ here entails that positive movements in the stock market influence positively short rates more than long rates. This makes the realization of negative spreads in combination with high stock market returns more likely, which again produces lower expected coupon payments. It is also observed that $\alpha$ is the most significant variable. From Table 1 , it is also clear that $\alpha$ is the most influential correlation parameter.

$\overline{15}$ In this implementation, the starting term structure is assumed flat and all the LIBOR rates $L_{i}(0)$ is assumed to have the same initial value.

${ }^{16}$ Or, in other words, a higher probabilty of observing a high stock return together with a high spread. 
Not all values of $\alpha$ and $\gamma$ are allowed in the simulation, since the correlation matrix (16) has to be positive semidefinite. This is the reason why the $\alpha$ and $\gamma$ axes in Figure 7 do not cover the same intervals as in Table 1.

\section{Conclusion}

In the paper, it was shown how the pricing of a new type of derivatives known as correlation/hybrid derivatives with payoffs depending on both a stock index and some LIBOR rates can be implemented in a LIBOR market model framework. The main advantage of using a LIBOR market framework is that the pricing is based on observable market quantities which makes the model easy to calibrate.

In some cases, a closed derivative pricing formula exists and the first contribution of this paper was the derivation of an analytically closed solution for the price of the traffic light option, when the underlying discounted stock price process and LIBOR rate are assumed lognormal under the forward measure. It was shown how the price of the derivative is a decreasing function of the initial value of the underlying variables, and it was illustrated how the correlation between the stock index and the LIBOR rate is of major importance. This is also the key obstacle when using Proposition 1, where all the parameters can be extracted from observed market data except for the correlation. The correlation between stocks and LIBOR rates is thus a natural research question to delve further into.

It was shown how the pricing of general correlation derivatives can be performed under the spot LIBOR measure. The dynamics of the stock index under this measure were derived, which to the knowledge of the author, is new. In many cases, pricing has to be done by Monte Carlo simulation, and the implementation of the model was illustrated via the pricing of a hybrid product known as the EUR Sage Note. Payments of this form are used extensively in the construction of structured products, and the approach described in this paper can be used for the pricing of these payoffs. 


\section{A Proof of Proposition 1}

PROOF. Under the forward measure $\mathbb{Q}^{n+1}$ conditioned on $\mathcal{F}_{t}$ both $\frac{S\left(T_{n+1}\right)}{B\left(T_{n+1}, T_{n+1}\right)}$ and $L_{n}\left(T_{n}\right)$ are lognormally distributed meaning that $\frac{S\left(T_{n+1}\right)}{B\left(T_{n+1}, T_{n+1}\right)}=\frac{S(t)}{B\left(t, T_{n+1}\right)} e^{X}$ and $L_{n}\left(T_{n}\right)=L_{n}(t) e^{Y}$, where $(X, Y)$ is bivariate normally distributed, independent of $\mathcal{F}_{t}$ and

$$
\left(\begin{array}{l}
X \\
Y
\end{array}\right) \sim N\left(\left(\begin{array}{l}
\mu_{x} \\
\mu_{y}
\end{array}\right),\left(\begin{array}{cc}
\sigma_{x}^{2} & \sigma_{x y} \\
\sigma_{x y} & \sigma_{y}^{2}
\end{array}\right)\right)
$$

with $\mu_{x}=-\frac{1}{2} \int_{t}^{T_{n+1}} \sigma_{s}^{2} d s, \mu_{y}=-\frac{1}{2} \int_{t}^{T_{n}} \lambda_{n}(s)^{2} d s, \sigma_{x}^{2}=\int_{t}^{T_{n+1}} \sigma_{s}^{2} d s$, $\sigma_{y}^{2}=\int_{t}^{T_{n}} \lambda_{n}(s)^{2} d s$ and $\sigma_{x y}=\int_{t}^{T_{n}} \sigma_{s} \lambda_{n}(s) \rho_{s} d s$.

Rewriting the expectation for the payoff (5) results in;

$$
\begin{aligned}
& B\left(t, T_{n+1}\right) \mathbb{E}^{n+1}\left[\left[\bar{S}-\frac{S(t)}{B\left(t, T_{n+1}\right)} e^{X}\right]^{+} \cdot\left[\bar{L}-L_{n}(t) e^{Y}\right]^{+} \mid \mathcal{F}_{t}\right] \\
& =S(t) L_{n}(t) \mathbb{E}^{n+1}\left[\left[\frac{\bar{S} \cdot B\left(t, T_{n+1}\right)}{S(t)}-e^{X}\right]^{+} \cdot\left[\frac{\bar{L}}{L_{n}(t)}-e^{Y}\right]^{+} \mid \mathcal{F}_{t}\right] \\
& =S(t) L_{n}(t) \mathbb{E}^{n+1}\left[\left[\widetilde{S}-e^{X}\right]^{+} \cdot\left[\widetilde{L}-e^{Y}\right]^{+} \mid \mathcal{F}_{t}\right] \\
& =S(t) L_{n}(t)\left\{\widetilde{S} \widetilde{L} \mathbb{E}^{n+1}\left[1_{\left\{e^{X}<\widetilde{S}\right\}} 1_{\left\{e^{Y}<\widetilde{L}\right\}} \mid \mathcal{F}_{t}\right]\right. \\
& -\widetilde{L} \mathbb{E}^{n+1}\left[e^{X} 1_{\left\{e^{X}<\widetilde{S}\right\}} 1_{\left\{e^{Y}<\widetilde{L}\right\}} \mid \mathcal{F}_{t}\right] \\
& \left.-\widetilde{S} \mathbb{E}^{n+1}\left[e^{Y} 1_{\left\{e^{X}<\widetilde{S}\right\}}\right\}_{\left\{e^{Y}<\widetilde{L}\right\}} \mid \mathcal{F}_{t}\right] \\
& \left.+\mathbb{E}^{n+1}\left[e^{X} e^{Y} 1_{\left\{e^{X}<\widetilde{S}\right\}} 1_{\left\{e^{Y}<\widetilde{L}\right\}} \mid \mathcal{F}_{t}\right]\right\} \\
& =S(t) L_{n}(t)\left\{\widetilde{S} \widetilde{L} \mathbb{E}^{n+1}\left[1_{\left\{e^{X}<\widetilde{S}\right\}^{1}}{ }_{\left\{e^{Y}<\widetilde{L}\right\}}\right]-\widetilde{L} \mathbb{E}^{n+1}\left[e^{X} 1_{\left\{e^{X}<\widetilde{S}\right\}} 1_{\left\{e^{Y}<\widetilde{L}\right\}}\right]\right. \\
& \left.-\widetilde{S} \mathbb{E}^{n+1}\left[e^{Y} 1_{\left\{e^{X}<\widetilde{S}\right\}} 1_{\left\{e^{Y}<\widetilde{L}\right\}}\right]+\mathbb{E}^{n+1}\left[e^{X} e^{Y} 1_{\left\{e^{X}<\widetilde{S}\right\}} 1_{\left\{e^{Y}<\widetilde{L}\right\}}\right]\right\} \text {. }
\end{aligned}
$$

The first equality is obvious, the third holds because $\widetilde{S}$ and $\widetilde{L}$ are both measurable with respect to $\mathcal{F}_{t}$ and the fourth holds because of the independence between the variables and the $\sigma$-algebra.

Finding the price of the traffic light option then reduces to evaluation of the four expectations in (A.1). Starting from the beginning:

(1) If $f$ denotes the density function for the standard bivariate normal dis- 
tribution, then the first expectation reduces to

$$
\begin{aligned}
& \mathbb{E}^{n+1}\left[1_{\left\{e^{X}<\widetilde{S}\right\}} 1_{\left\{e^{Y}<\widetilde{L}\right\}}\right] \\
& =\mathbb{E}^{n+1}\left[1_{\{X<\ln \widetilde{S}\}} 1_{\{Y<\ln \widetilde{L}\}}\right] \\
& =\int_{-\infty}^{\ln \widetilde{S}} \int_{-\infty}^{\ln \widetilde{L}} f(x, y) d y d x \\
& =\int_{-\infty}^{\ln \widetilde{S}} \int_{-\infty}^{\ln \widetilde{L}} \frac{1}{2 \pi \sigma_{x} \sigma_{y} \sqrt{1-\rho^{2}}} \\
& \times \exp \left\{-\frac{1}{2\left(1-\rho^{2}\right)}\right. \\
& \left.\times\left[\left(\frac{x-\mu_{x}}{\sigma_{x}}\right)^{2}-2 \rho\left(\frac{x-\mu_{x}}{\sigma_{x}}\right)\left(\frac{y-\mu_{y}}{\sigma_{y}}\right)+\left(\frac{y-\mu_{y}}{\sigma_{y}}\right)^{2}\right]\right\} d y d x \\
& =M\left(\frac{\ln \widetilde{S}-\mu_{x}}{\sigma_{x}}, \frac{\ln \widetilde{L}-\mu_{y}}{\sigma_{y}} ; \rho_{S L}\right),
\end{aligned}
$$

where the fourth equality follows by substituting $u=\frac{x-\mu_{x}}{\sigma_{x}}$ and $v=\frac{y-\mu_{y}}{\sigma_{y}}$.

$$
\mathbb{E}^{n+1}\left[e^{X} 1_{\left\{e^{X}<\widetilde{S}\right\}} 1_{\left\{e^{Y}<\widetilde{L}\right\}}\right]=\int_{-\infty}^{\ln \widetilde{L}} \int_{-\infty}^{\ln \widetilde{S}} e^{x} f(y) f(x \mid y) d x d y
$$

Rewriting the exponent gives

$$
\begin{aligned}
& x-\frac{1}{2}\left(\frac{y-\mu_{y}}{\sigma_{y}}\right)^{2}-\frac{1}{2 \sigma_{x}^{2}\left(1-\rho^{2}\right)}\left(x-\mu_{x}-\frac{\rho \sigma_{x}}{\sigma_{y}}\left(y-\mu_{y}\right)\right)^{2} \\
& =\mu_{x}+\frac{1}{2} \sigma_{x}^{2}-\frac{1}{2\left(1-\rho^{2}\right)}\left(u^{2}-2 \rho u v+v^{2}\right)
\end{aligned}
$$

where the substitutions $u=\frac{x-\mu_{x}}{\sigma_{x}}-\sigma_{x}$ and $v=\frac{y-\mu_{y}}{\sigma_{y}}-\rho \sigma_{x}$ have been made. This again gives

$$
\begin{aligned}
& \mathbb{E}^{n+1}\left[e^{X} 1_{\left\{e^{X}<\widetilde{S}\right\}} 1_{\left\{e^{Y}<\widetilde{L}\right\}}\right] \\
& =e^{\mu_{x}+\frac{1}{2} \sigma_{x}^{2}} \cdot M\left(\frac{\ln \widetilde{S}-\mu_{x}}{\sigma_{x}}-\sigma_{x}, \frac{\ln \widetilde{L}-\mu_{y}}{\sigma_{y}}-\rho_{S L} \sigma_{x} ; \rho_{S L}\right) .
\end{aligned}
$$

(3) The third expectation can be calculated in the same way as the second.

$$
\begin{aligned}
& \mathbb{E}^{n+1}\left[e^{Y} 1_{\left\{e^{X}<\widetilde{S}\right\}} 1_{\left\{e^{Y}<\widetilde{L}\right\}}\right] \\
& =e^{\mu_{y}+\frac{1}{2} \sigma_{y}^{2}} \cdot M\left(\frac{\ln \widetilde{S}-\mu_{x}}{\sigma_{x}}-\rho_{S L} \sigma_{y}, \frac{\ln \widetilde{L}-\mu_{y}}{\sigma_{y}}-\sigma_{y} ; \rho_{S L}\right) .
\end{aligned}
$$


$(4)$

$$
\mathbb{E}^{n+1}\left[e^{X} e^{Y} 1_{\left\{e^{X}<\widetilde{S}\right\}} 1_{\left\{e^{Y}<\widetilde{L}\right\}}\right]=\int_{-\infty}^{\ln \widetilde{S}} \int_{-\infty}^{\ln \widetilde{L}} e^{x} e^{y} f(x) f(y \mid x) d y d x
$$

Rewriting the exponent again gives

$$
\begin{aligned}
& x+y-\frac{1}{2}\left(\frac{x-\mu_{x}}{\sigma_{x}}\right)^{2}-\frac{1}{2 \sigma_{y}^{2}\left(1-\rho^{2}\right)}\left(y-\mu_{y}-\frac{\rho \sigma_{y}}{\sigma_{x}}\left(x-\mu_{x}\right)\right)^{2} \\
& =\mu_{x}+\mu_{y}+\frac{1}{2} \sigma_{x}^{2}+\frac{1}{2} \sigma_{y}^{2}+\rho \sigma_{x} \sigma_{y}-\frac{1}{2\left(1-\rho^{2}\right)}\left(u^{2}-2 \rho u v+v^{2}\right),
\end{aligned}
$$

where the substitutions $u=\frac{x-\mu_{x}}{\sigma_{x}}-\rho \sigma_{y}-\sigma_{x}$ and $v=\frac{y-\mu_{y}}{\sigma_{y}}-\rho \sigma_{x}-\sigma_{y}$ have been made. Together the above finally results in

$$
\begin{aligned}
& \mathbb{E}^{n+1}\left[e^{X} e^{Y} 1_{\left\{e^{X}<\widetilde{S}\right\}} 1_{\left\{e^{Y}<\widetilde{L}\right\}}\right] \\
& =e^{\mu_{x}+\mu_{y}+\frac{1}{2} \sigma_{x}^{2}+\frac{1}{2} \sigma_{y}^{2}+\rho \sigma_{x} \sigma_{y}} \times \\
& M\left(\frac{\ln \widetilde{S}-\mu_{x}}{\sigma_{x}}-\rho_{S L} \sigma_{y}-\sigma_{x}, \frac{\ln \widetilde{L}-\mu_{y}}{\sigma_{y}}-\rho_{S L} \sigma_{x}-\sigma_{y} ; \rho_{S L}\right) .
\end{aligned}
$$

Now collecting all the terms, and observing that for our particular claim $\mu_{x}=-\frac{1}{2} \sigma_{x}^{2}$ and $\mu_{y}=-\frac{1}{2} \sigma_{y}^{2}$, the desired result is achieved. 


\section{References}

[1] Björk, T. (2004). Arbitrage Theory in Continuous Time, second edition, Oxford University Press.

[2] Black, F. (1976). The Pricing of Commodity Contracts, Journal of Financial Economics 3, pp. 167-179.

[3] BNP Paribas (2006). Derivatives Handbook 2006.

[4] Brace, A. Gatarek, D. and Musiela, M. (1997). The Market Model of Interest Rate Dynamics, Mathematical Finance 7, pp. 127-155.

[5] Brigo, D. and Mercurio, F. (2006). Interest Rate Models - Theory and Practice, second edition, Springer.

[6] Delbaen, F. and Schachermayer, W. (1998). The Fundamental Theorem of Asset Pricing for Unbounded Stochastic Processes, Math. Ann. 312, pp. 215-250.

[7] Hunter, C. and Picot, G. (2005). Hybrid Derivatives - Financial Engines of the Future, The Euromoney Derivatives and Risk Management Handbook 2005/2006, Chapter 8, Euromoney Yearbooks.

[8] Glasserman, P. (2004). Monte Carlo Methods in Financial Engineering, Springer.

[9] Glasserman, P. and Zhao, X. (2000). Arbitrage-Free Discretization of Lognormal Forward LIBOR and Swap Rate Models, Finance and Stochastics 4, pp. 35-68.

[10] Hull, J. and White, A. (1999). Forward Rate Volatilities, Swap Rate Volatilities, and the Implementation of the LIBOR Market Model, Journal of Fixed Income 10, pp. 46-62.

[11] Jamshidian, F. (1997). LIBOR and Swap Market Models and Measures, Finance and Stochastics 1, pp. 293-330.

[12] Jørgensen, P. L. (2007). Traffic Light Options, Journal of Banking and Finance 31, pp. 3698-3719 .

[13] Miltersen, K. Sandmann, K. and Sondermann, D. (1997). Closed Form Solutions for Term Structure Derivatives with Lognormal Interest Rates, Journal of Finance 52, pp. 409-430.

[14] Musiela, M. and Rutkowski, M. (2004). Martingale Methods in Financial Modelling, second edition., Springer.

[15] Rebonato, R. (2002). Modern Pricing of Interest-Rate-Derivatives, Princeton University Press. 


\section{Working Papers from Finance Research Group}

F-2008-01 Thomas Kokholm: Pricing of Traffic Light Options and other Correlation Derivatives.

F-2007-03 Domenico De Giovanni: Lapse Rate Modeling: A Rational Expectation Approach.

F-2007-02 Andrea Consiglio \& Domenico De Giovanni: Pricing the Option to Surrender in Incomplete Markets.

F-2006-09 Peter Løchte Jørgensen: Lognormal Approximation of Complex Pathdependent Pension Scheme Payoffs.

F-2006-08 Peter Løchte Jørgensen: Traffic Light Options.

F-2006-07 David C. Porter, Carsten Tanggaard, Daniel G. Weaver \& Wei Yu: Dispersed Trading and the Prevention of Market Failure: The Case of the Copenhagen Stock Exhange.

F-2006-06 Amber Anand, Carsten Tanggaard \& Daniel G. Weaver: Paying for Market Quality.

F-2006-05 Anne-Sofie Reng Rasmussen: How well do financial and macroeconomic variables predict stock returns: Time-series and cross-sectional evidence.

F-2006-04 Anne-Sofie Reng Rasmussen: Improving the asset pricing ability of the Consumption-Capital Asset Pricing Model.

F-2006-03 Jan Bartholdy, Dennis Olson \& Paula Peare: Conducting event studies on a small stock exchange.

F-2006-02 Jan Bartholdy \& Cesário Mateus: Debt and Taxes: Evidence from bankfinanced unlisted firms.

F-2006-01 Esben P. Høg \& Per H. Frederiksen: The Fractional Ornstein-Uhlenbeck Process: Term Structure Theory and Application.

F-2005-05 Charlotte Christiansen \& Angelo Ranaldo: Realized bond-stock correlation: macroeconomic announcement effects.

F-2005-04 Søren Willemann: GSE funding advantages and mortgagor benefits:

Answers from asset pricing.

F-2005-03 Charlotte Christiansen: Level-ARCH short rate models with regime switching: Bivariate modeling of US and European short rates.

F-2005-02 Charlotte Christiansen, Juanna Schröter Joensen and Jesper Rangvid: Do more economists hold stocks? 
F-2005-01 Michael Christensen: Danish mutual fund performance - selectivity, market timing and persistence.

F-2004-01 Charlotte Christiansen: Decomposing European bond and equity volatility. 
ISBN 87-7882-177-0

Department of Business Studies

Aarhus School of Business

University of Aarhus

Fuglesangs Allé 4

DK-8210 Aarhus V - Denmark

Tel. +4589486688

Fax +4586150188

www.asb.dk 